\title{
A PDE-BASED METHODOLOGY FOR MODELING, PARAMETER ESTIMATION AND FEEDBACK CONTROL IN STRUCTURAL AND STRUCTURAL ACOUSTIC SYSTEMS ${ }^{1}$
}

\author{
H.T. Banks \\ Center for Research in Scientific Computation \\ North Carolina State University \\ Raleigh, NC 27695 \\ D.E. Brown \\ Lockheed Engineering and Sciences Company \\ NASA Langley Research Center \\ Hampton, VA 23681 \\ Vern L. Metcalf \\ U.S. Army Research Laboratory \\ NASA Langley Research Center \\ Hampton, VA 23681 \\ R.J. Silcox \\ Acoustics Division \\ NASA Langley Research Center \\ Hampton, VA 23681 \\ R.C. Smith \\ Department of Mathematics \\ Iowa State University \\ Ames, IA 50011 \\ Yun Wang \\ Center for Research in Scientific Computation \\ North Carolina State University \\ Raleigh, NC 27695
}

\begin{abstract}
A problem of continued interest concerns the control of vibrations in a flexible structure and the related problem of reducing structure-borne noise in structural acoustic systems. In both cases, piezoceramic patches bonded to the structures have been successfully used as control actuators. Through the application of a controlling voltage, the patches can be used to reduce structural vibrations which in turn leads to methods for reducing structure-borne noise. A PDE-based methodology for modeling, estimating physical parameters, and implementing a feedback control scheme for problems of this type is discussed. While the illustrating example is a circular plate, the methodology is sufficiently general so as to be applicable in a variety of structural and structural acoustic systems.
\end{abstract}

\footnotetext{
${ }^{1}$ The research of H.T.B. and Y.W. was supported in part by the Air Force Office of Scientific Research under grant AFOSR-F4962093-1-0198. This research was also supported by the National Aeronautics and Space Administration under NASA Contract Numbers NAS1-18605 and NAS1-19480 while H.T.B., R.C.S. and Y.W. were visiting scientists at the Institute for Computer Applications in Science and Engineering (ICASE), NASA Langley Research Center, Hampton, VA 23681. Finally, the research of D.E.B. was supported under NASA Contract Number NAS1-19000.
} 


\section{INTRODUCTION}

In recent years, a great deal of work has been done on the development of control methodologies for structural and structural acoustic applications using piezoelectric actuators. One such type of actuator consists of piezoceramic patches which are bonded to the structure in the system of interest. Through the input of voltage to the patches, in-plane forces and/or bending moments can be created which affect the structural motion and can ultimately be used to reduce vibration levels or control structure-borne noise.

One method of determining the manner through which the piezoceramic patches can be incorporated as controlling elements in structural and structural acoustic systems is through distributed parameter models [1, 2, 3, 4, 5]. Alternative control approaches using piezoceramic patches for similar problems are illustrated in $[6,7,8,9,10]$. In distributed parameter approaches, one typically models the physics of the system under investigation by a partial differential equation (PDE) or system of PDE's at which point, infinite dimensional parameter estimation and control results can be obtained. Corresponding finite dimensional approximations result from the discretization of the PDE's. To date, numerous theoretical results concerning the modeling, estimation of physical parameters and feedback control in structural and structural acoustic systems have been obtained with supporting numerical results demonstrating the feasibility of the methods in 2-D and 3-D geometries (see $[14,15]$ for modeling results for a 3 -D structural acoustic system currently under investigation in the Acoustics Division, NASA Langley Research Center, and [2, 5] for numerical examples demonstrating the PDE-based techniques for a model involving a 2-D slice of this setup). The experimental success of the PDE-based parameter estimation techniques has also been demonstrated in studies involving various flexible structures including beams [11, 12] and multiple component structures [13]. However, little experimental work has been done on the estimation of physical parameters in more complex structures or structural acoustic systems, and the PDE-based control techniques involving piezoceramic actuators have not previously been implemented.

This paper contains preliminary results obtained when distributed parameter techniques for parameter estimation and feedback control were experimentally implemented on a circular plate. These results are intended to illustrate techniques for implementing parameter estimation and feedback control through proof-of-concept experiments with an isolated component from the structural acoustic system described in $[14,15]$ (the structure in that system is made up of a hardwalled cylinder with a clamped circular plate at one end, and the control problem consists of using piezoceramic patches on the plate to reduce the interior structure-borne sound pressure levels which result when the plate is subjected to a strong exterior acoustic field). In addition to providing a preliminary test regarding the experimental implementation of the PDE-based method for reducing structural vibrations, this also provides a first step toward a feasible experimental implementation of the theoretical PDE techniques for full structural acoustic systems.

\section{MATHEMATICAL MODEL}

In this section, a brief discussion of the mathematical model used to describe the experimental setup is given and the approximation techniques used to discretize the model are outlined. To simplify the presentation, a uniform circular plate, clamped around its perimeter and subjected to an axisymmetric exciting force $g(t, r)$, is considered (it is emphasized, however, that all of the results and techniques presented here extend directly to the more general case of nonaxisymmetric excitation and vibrations). Bonded to the plate is a centrally placed piezoceramic circular patch which is used to damp the plate vibrations when a controlling voltage is applied (see Figure 1).
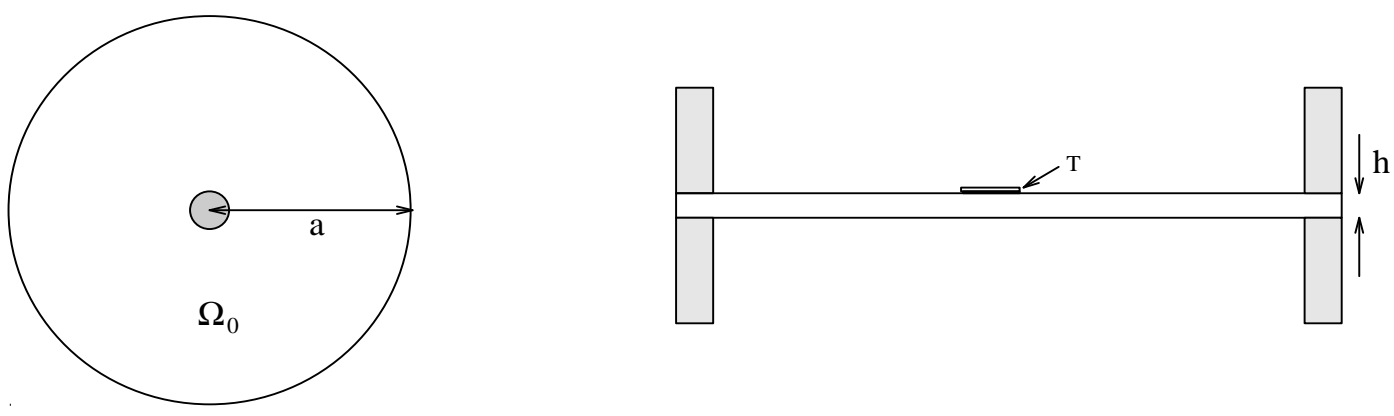

Fig. 1. Clamped circular plate of radius $a$ and thickness $h$ with a centered circular piezoceramic patch of thickness $T$. 
For a thin circular plate of radius $a$, thickness $h$, and occupying the region $\Omega_{0}$, the weak or variational (energy) form of the axisymmetric equations of pure bending motion is

$$
\begin{gathered}
\int_{\Omega_{0}} \rho h w_{t t} \bar{\eta} d \omega+\int_{\Omega_{0}} \gamma w_{t} \bar{\eta} d \omega+\int_{\Omega_{0}} M_{r} \overline{\eta_{r r}} d \omega+\int_{\Omega_{0}} \frac{1}{r} M_{\theta} \overline{\eta_{r}} d \omega \\
=\int_{\text {patch }} \mathcal{K}^{B} u(t) \overline{\nabla^{2} \eta} d \omega+\int_{\Omega_{0}} g \bar{\eta} d \omega
\end{gathered}
$$

for all functions $\eta \in V$ (note that $d \omega=r d r d \theta$ and the overbars denote complex conjugation). Here $w$ is the transverse plate displacement, $\rho$ is the plate density, $\gamma$ denotes the viscous damping coefficient, $u(t)$ is the voltage applied to the patch, and $V$ (the space of test functions) is the subset of the Sobolev space $H^{2}\left(\Omega_{0}\right)$ satisfying the fixed boundary conditions $w=w_{r}=0$ at $r=a$. The internal bending moments are given by

$$
\begin{aligned}
& M_{r}=D\left(\frac{\partial^{2} w}{\partial r^{2}}+\frac{\nu}{r} \frac{\partial w}{\partial r}\right)+c_{D}\left(\frac{\partial^{3} w}{\partial r^{2} \partial t}+\frac{\nu}{r} \frac{\partial^{2} w}{\partial r \partial t}\right) \\
& M_{\theta}=D\left(\frac{1}{r} \frac{\partial w}{\partial r}+\nu \frac{\partial^{2} w}{\partial r^{2}}\right)+c_{D}\left(\frac{1}{r} \frac{\partial^{2} w}{\partial r \partial t}+\nu \frac{\partial^{3} w}{\partial r^{2} \partial t}\right) .
\end{aligned}
$$

With $E$ denoting the Young's modulus, the parameters $D=\frac{E h^{3}}{12\left(1-\nu^{2}\right)}, c_{D}$ and $\nu$ represent the flexural rigidity, KelvinVoigt damping coefficient and Poisson's ratio for the plate/patch structure. We point out that $\rho, D, \nu$ and $c_{D}$ are assumed to be piecewise constant to account for the change in material properties and thickness in the region occupied by the patch (see [12] for the experimentally verified necessity of such an assumption). We also note that the KelvinVoigt and viscous terms are used to model the natural damping of the plate motion as it vibrates. Finally, as discussed in [16], the constant

$$
\mathcal{K}^{B}=\frac{1}{2} \frac{E_{p e}}{1-\nu_{p e}} d_{31}\left(h+2 T_{b \ell}+T\right)
$$

results from the integration of the free stresses generated by the patch (here, $E_{p e}, T, \nu_{p e}, d_{31}$ are the Young's modulus, thickness, Poisson ratio, and piezoelectric strain constant for the patch while $T_{b \ell}$ denotes the bonding layer thickness). Summarizing, the plate equation (1) contains (in order from left to right) an inertial term, a viscous damping contribution, internal bending components which incorporate the stiffness and Kelvin-Voigt damping for the structure, the external moment due to application of voltage to the patch, and the forcing term due to the exterior exciting force $g$.

To approximate the plate dynamics, a Fourier-Galerkin scheme is used to discretize the infinite dimensional system (1). As detailed in [15], the plate displacement is approximated by

$$
w^{N}(t, r, \theta)=\sum_{n=1}^{N} w_{n}^{N}(t) B_{n}(r, \theta)
$$

where $B_{n}(r, \theta)$ are cubic spline/Fourier basis functions. The substitution of the expansion (3) into (1) with orthogonalization against basis elements yields the $2 N \times 2 N$ matrix system

$$
\begin{aligned}
& M^{N} \dot{y}^{N}(t)=\tilde{A}^{N} y^{N}(t)+\tilde{B}^{N} u(t)+\tilde{G}^{N}(t) \\
& M^{N} y^{N}(0)=\tilde{y}_{0}^{N}
\end{aligned}
$$

or equivalently, the Cauchy system

$$
\begin{aligned}
& \dot{y}^{N}(t)=A^{N} y^{N}(t)+B^{N} u(t)+G^{N}(t) \\
& y^{N}(0)=y_{0}^{N}
\end{aligned}
$$

where $y^{N}(t)=\left[w_{1}^{N}(t), \cdots, w_{N}^{N}(t), \dot{w}_{1}^{N}(t), \cdots, \dot{w}_{N}^{N}(t)\right]$ denotes the $2 N \times 1$ vector containing the generalized Fourier coefficients for the approximate displacement and velocity (again, see [15] for details concerning the discretization of the circular plate equation and formulation of the matrices $A^{N}, B^{N}$ and $G^{N}$ ). In this form, the finite dimensional parameter estimation and control problems can be discussed. 


\section{PARAMETER ESTIMATION}

The goal of the finite dimensional parameter estimation problem is to determine estimates of the "true" material parameters $\rho, \gamma, D, \nu, c_{D}$ and $\mathcal{K}^{B}$ given data measurements $z$ (in the experimental results reported here, the data was a time history of the normal acceleration obtained from an accelerometer mounted at the center of the plate). Letting $q=\left(\rho, \gamma, D, \nu, c_{D}, \mathcal{K}^{B}\right)$ and assuming that $q \in Q$ where $Q$ denotes an admissible parameter space, the finite dimensional parameter estimation problem is to then seek $\bar{q} \in Q$ which minimizes

$$
J^{N}(q)=\sum_{i}\left|\frac{\partial^{2} w^{N}}{\partial t^{2}}\left(t_{i}, \bar{r}, \bar{\theta} ; q\right)-z_{i}\right|^{2}
$$

subject to $w^{N}$ satisfying the approximating coupled system equations (hence the coefficients $\left\{w_{n}^{N}(t)\right\}$ of $w^{N}$ must satisfy (4)). In this case, we have $(\bar{r}, \bar{\theta})=(0,0)$ since the accelerometer is located at the center of the plate. As indicated previously, the material parameters $\rho, D, \nu$ and $c_{D}$ are assumed to be piecewise constant in order to account for the differing structural properties in the region of the plate covered by the patch.

The dimensions of the aluminum plate and piezoceramic patch are summarized in Table 1. This table also contains "handbook" values for the Young's modulus, Poisson ratio and density of the plate and patch. We reiterate that while the "handbook" values provide a starting point in the parameter estimation routine, they usually cannot be used in the final system model with any accuracy due to nonuniformities in the plate or boundary conditions, variations in materials, and the contributions due to the presence of the patch.

The following strategy was used to estimate the parameters for the experimental plate. An impact hammer hit to the center of the plate was used to excite the structure and data was collected from a centered accelerometer on the opposite side of the plate. The excitation of the structure in this manner provided a primarily axisymmetric response with the purely axisymmetric component being measured by the accelerometer. Data obtained from off-center accelerometers indicated that while slight nonaxisymmetric vibrations were present, their effect was minimal.

With a time history of the acceleration data, the minimization of (5) was performed subject to $w^{N}$ satisfying the discretization of the PDE-based plate model. The estimated parameters $\rho, \gamma, D, \nu$ and $c_{D}$ are recorded in Table 2 and model-based results obtained with these values are plotted against experimental results in Figures 2 and 3 . As indicated by the results in Figure 3, four axisymmetric modes were excited with the primary response being in the first two modes having frequencies of 57.9 hertz and 224.9 hertz, respectively. The results in both figures demonstrate that the parameter estimates in Table 2 lead to a very close matching of the first two frequencies (the response of the third axisymmetric mode is too weak to permit adequate comparison). The overdamping of the high frequency mode is characteristic of the Kelvin-Voigt damping model and this leads to the very slight variation seen in the time history when comparing the experimental data and model response.

In order to obtain the patch parameter $\mathcal{K}_{B}$, the system was excited via a voltage spike to the patch, acceleration data was collected, and the above process was repeated (we point out that this technique alone can be used to estimate all of the parameters but with a response that contains much higher frequencies than those excited by the hammer). In comparing the estimated value of $\mathcal{K}_{B}$ with that calculated from the expression (2), one notes an approximately $13 \%$ difference in values (to calculate a value for $\mathcal{K}_{B}$, a bonding layer of thickness $T_{b \ell}=T / 5$ was assumed). This falls well within the $20 \%$ variation that is often associated with the "handbook" value of $d_{31}$. The estimated values in Table 2 can then be used in the control method described in the next section.

\begin{tabular}{l|l|l} 
& Plate Properties & Patch Properties \\
\hline Radius & $a=.2286 \mathrm{~m}\left(9^{\prime \prime}\right)$ & $\mathrm{rad}=.01905 \mathrm{~m}\left(.75^{\prime \prime}\right)$ \\
Thickness & $h=.00127 \mathrm{~m}\left(.05^{\prime \prime}\right)$ & $T=.0001778 \mathrm{~m}\left(.007^{\prime \prime}\right)$ \\
Young's Modulus & $E=7.1 \times 10^{10} \mathrm{~N} / \mathrm{m}^{2}$ & $E_{p e}=6.3 \times 10^{10} \mathrm{~N} / \mathrm{m}^{2}$ \\
Density & $\rho=2700 \mathrm{~kg} / \mathrm{m}^{3}$ & $\rho_{p e}=7600 \mathrm{~kg} / \mathrm{m}^{3}$ \\
Poisson ratio & $\nu=.33$ & $\nu_{p e}=.31$ \\
Strain Coefficient & & $d_{31}=190 \times 10^{-12} \mathrm{~m} / \mathrm{V}$ \\
\hline
\end{tabular}

Table 1. Dimensions and "handbook" characteristics of the plate and PZT piezoceramic patch. 


\begin{tabular}{c|c|c|c|c}
\hline \multicolumn{2}{l|}{} & Analytic & Experimental & \% Variation \\
\hline $\begin{array}{c}\rho \cdot \text { thickness } \\
\left(\mathrm{kg} / \mathrm{m}^{2}\right)\end{array}$ & Plate & 3.429 & 3.157 & 8.6 \\
\hline$D$ & Plate + Pzt & & 3.123 & \\
\hline$(N \cdot m)$ & Plate + Pzt & 13.601 & 11.017 & 23.4 \\
\hline$c_{D}$ & Plate & & $2.158-4$ & \\
$(N \cdot m \cdot s e c)$ & Plate + Pzt & & $2.210-4$ & \\
\hline$\nu$ & Plate & .33 & .3304 & 0.1 \\
\hline & Plate + Pzt & & .3271 & \\
\hline \multicolumn{2}{c|}{$(\mathrm{sec} \cdot N / m)$} & & 15.566 & \\
\hline \multicolumn{2}{c|}{$\mathcal{K}^{B}(N / V)$} & .013369 & .015288 & 12.6 \\
\hline
\end{tabular}

Table 2. Analytic and experimental values of the physical parameters.

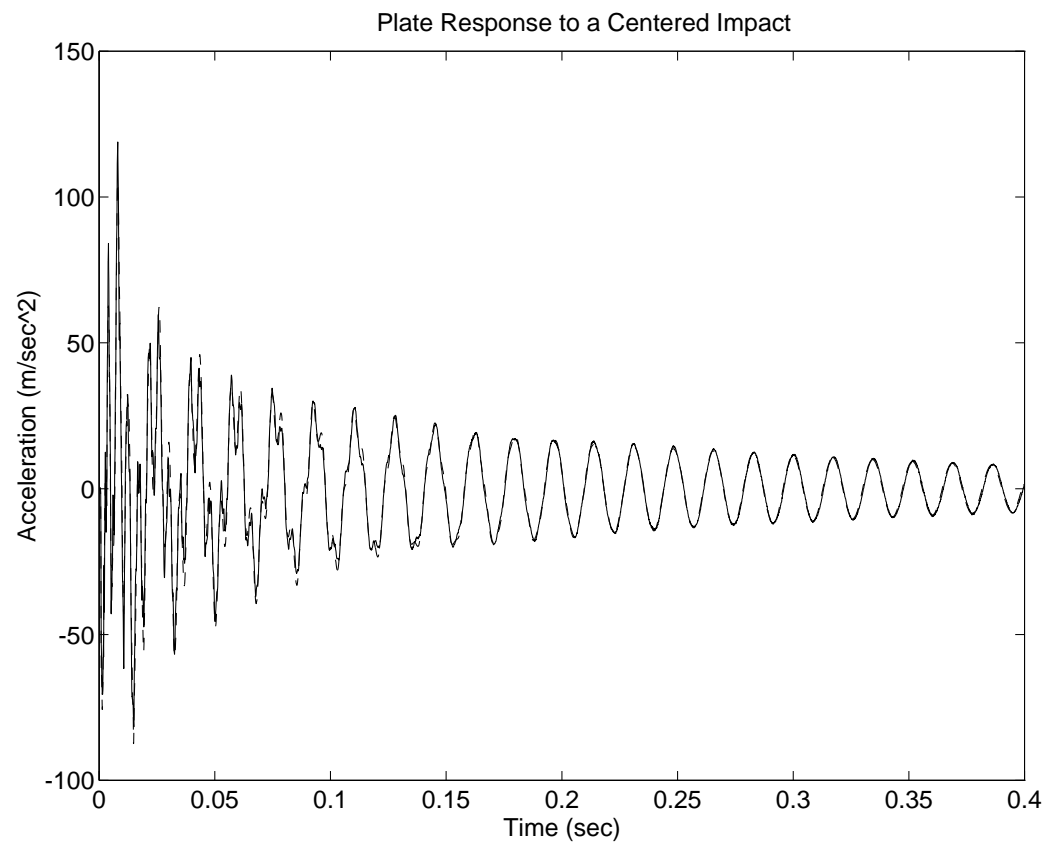

Fig. 2. Time history of the experimental data and model response with estimated parameters, -__ (Experimental Data), - - - (Model Response). 


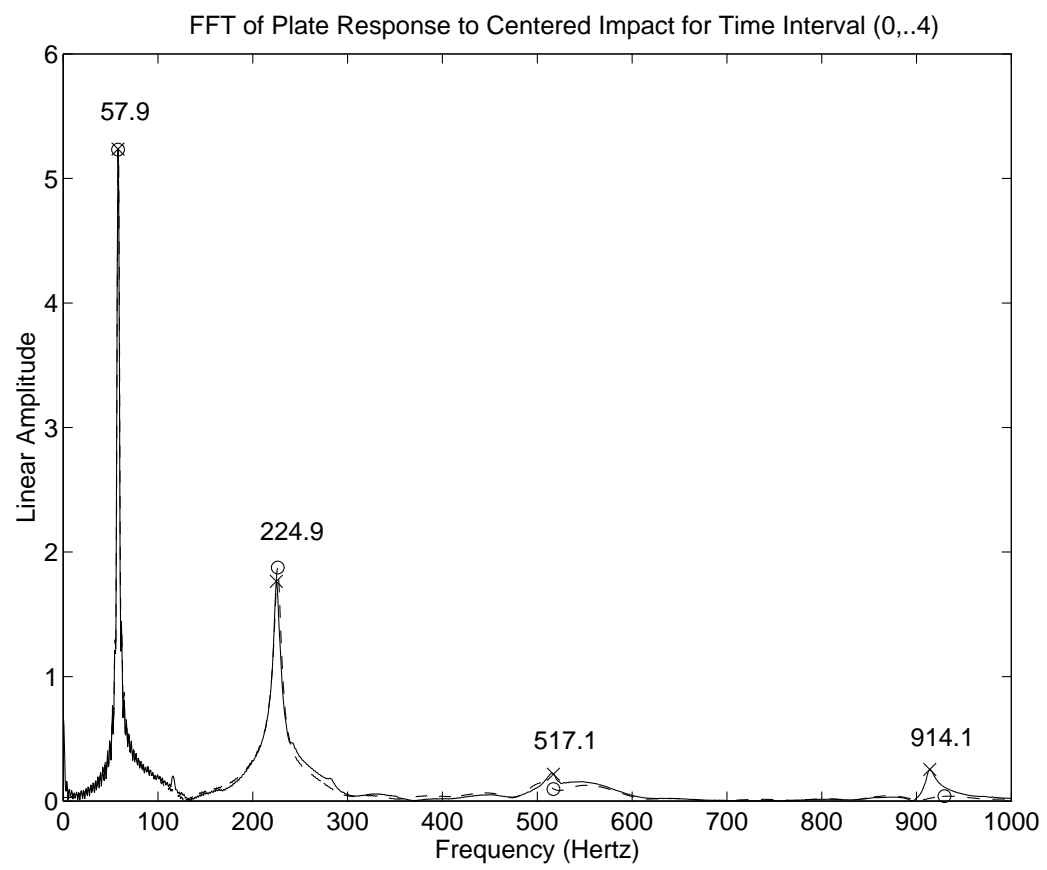

Fig. 3. Frequency content of the experimental data and thin plate model with estimated parameters, $\mathrm{x}-$ (Experimental Data), o - - (Model Response).

\section{VIBRATION CONTROL}

In considering the control problem, we will concentrate on the case in which the plate starts with an initial displacement and velocity and is then allowed to vibrate until motion stops. It is also assumed that the only external moments and forces being applied to the plate are the moments generated by the patch in response to an applied voltage which implies that $G^{N}(t)=0$ in the approximate system equation (4). The goal in the control problem is to determine a voltage $u(t)$ which, when applied to the piezoceramic patch, leads to a reduced level of vibration.

\subsection{Output Feedback Control}

For the finite dimensional approximate system, the problem of determining a controlling voltage can be posed as the problem of finding $u$ which minimizes

$$
J^{N}(u)=\int_{0}^{\infty}\left\{\left\langle Q^{N} y^{N}(t), y^{N}(t)\right\rangle_{\mathbb{R}^{2 N}}+\langle R u(t), u(t)\rangle_{\mathbb{R}}\right\} d t
$$

where $y^{N}(t)$ solves (4) and $R$ is a weight which penalizes overly large voltages (this ensures that realistic voltages are determined). As seen in [2], energy considerations suggest that a diagonal matrix multiple of the mass matrix $M^{N}$ is an appropriate choice for the nonnegative matrix $Q^{N}$. Under suitable conditions, optimal control theory can then be used to show that the optimal controlling voltage is given by

$$
u(t)=-K^{N} y^{N}(t)
$$

where $K^{N}=R^{-1}\left(B^{N}\right)^{T} \Pi^{N}$ and $\Pi^{N}$ is the solution to the algebraic Riccati equation

$$
\left(A^{N}\right)^{T} \Pi^{N}+\Pi^{N} A^{N}-\Pi^{N} B^{N} R^{-1}\left(B^{N}\right)^{T} \Pi^{N}+Q^{N}=0 .
$$

We remind the reader that in this finite dimensional formulation, $y^{N}(t)$ is a $2 N \times 1$ vector which contains the generalized Fourier coefficients $\left\{w_{n}^{N}(t)\right\}$ and $\left\{\dot{w}_{n}^{N}(t)\right\}$ for the approximate plate displacement and velocity (see (3)). 
A disadvantage to the feedback law (6) is that it requires knowledge of the displacement and velocity everywhere on the plate in order to be implemented. In reality, however, one usually is able to measure data only at a small number of locations on the plate and from these values, one must reconstruct (e.g., see [17]) or estimate the state (displacement and velocity) before using it to determine the controlling voltage. This yields a feedback law of the form

$$
u(t)=-K^{N} y_{c}^{N}(t)
$$

where $y_{c}^{N}(t)$ solves the state estimator or compensator equation

$$
\begin{aligned}
& \dot{y}_{c}^{N}(t)=\left[A^{N}-F^{N} C^{N}-B^{N} K^{N}\right] y_{c}^{N}(t)+F^{N} y_{o b}^{N}(t) \\
& y_{c}^{N}(0)=y_{c 0}^{N}
\end{aligned}
$$

(compare to the original finite dimensional system (4) with the expression (7) for the voltage). Here $C^{N}$ is a matrix specifying the points where displacement or velocity data is observed. The matrix $F^{N}$ has a form similar to that of the gain $K^{N}$ and is given by $F^{N}=P^{N}\left(C^{N}\right)^{T} N_{e}^{-1}$ where $N_{e}$ is a design matrix which is related to the effect of noise in the data (this is the "optimal" state estimator in the case of certain types of noise in the state and observation equations), and $P^{N}$ solves a second algebraic Riccati equation

$$
A^{N} P^{N}+P^{N}\left(A^{N}\right)^{T}-P^{N}\left(C^{N}\right)^{T} N_{e}^{-1} C^{N} P^{N}+M_{e}^{N}=0
$$

(the matrix $M_{e}^{N}$ is also a noise-related design parameter). The vector $y_{o b}^{N}(t)$ contains the generalized Fourier coefficients for the observed state (in this case, observed at the origin) and it is from this information that the state is approximated.

\subsection{Experimental Implementation}

As mentioned in the last section, data for these experiments was obtained with a centered accelerometer, and the first step in the algorithm for determining the controlling voltage at time $t_{k}$ was to integrate the accelerometer data to obtain velocity values. This step is very sensitive to the presence of DC gains in the data processing and was accomplished using the second order filter described in [18]. After projecting the velocity values into the finite dimensional subspace in which the dynamics are being approximated to obtain $y_{o b}^{N}$, the solution to (8) was stepped from time $t_{k-1}$ to $t_{k}$ to obtain the state estimate $y_{c}^{N}\left(t_{k}\right)$. This value was then multiplied by the gain $K^{N}$ to produce the controlling voltage $u\left(t_{k}\right)$ to be fed back into the system. By computing the gains and component matrices offline, the voltages could be calculated in real time thus permitting this feedback method to be implemented experimentally.

As a preliminary test into the feasibility of implementing this feedback control scheme, a series of experiments were conducted in which the plate was excited with a centered impact hammer strike and the vibrations were recorded both with and without controlling voltages being applied. In all cases, the same electronic setup was used with the control cases differing only in that the calculated voltage ( 7 ) was fed back into the system whereas it was simply calculated and stored in the uncontrolled cases. Representative plots of the plate velocity (integrated from the data recorded by the centered accelerometer) for the uncontrolled and controlled cases are given in Figure 4. The voltage (from (7)) yielding the controlled results is plotted in Figure 5. Finally, the force delivered by the hammer impact in the uncontrolled and controlled cases is recorded in Figure 6. As indicated by the results in this latter figure, the force delivered in the two cases was nearly identical (a slight double hit was always present when an impact hammer was used to excite the axisymmetric modes). By comparing the velocity levels in the two cases, it is noted that a significant reduction was obtained in the controlled case with a controlling voltage having a maximum value below $60 \mathrm{~V}$. Quantitatively, the application of the controlling voltage leads to a $47 \%$ reduction in maximum velocity levels by time $T=.5 \mathrm{sec}$ and a reduction of $73 \%$ by $T=1$ sec.

We point out that these results are typical of those recorded in a series of experiments and hence represent the typical reductions in vibration levels that were obtained when the controlling voltage was fed back into the system in the manner described above. While implementation procedures are not yet optimal, these preliminary tests demonstrated that vibration levels could be effectively reduced when the PDE-based control scheme was implemented, and they indicate directions for future experimental tests to further improve the technique and methodology. 


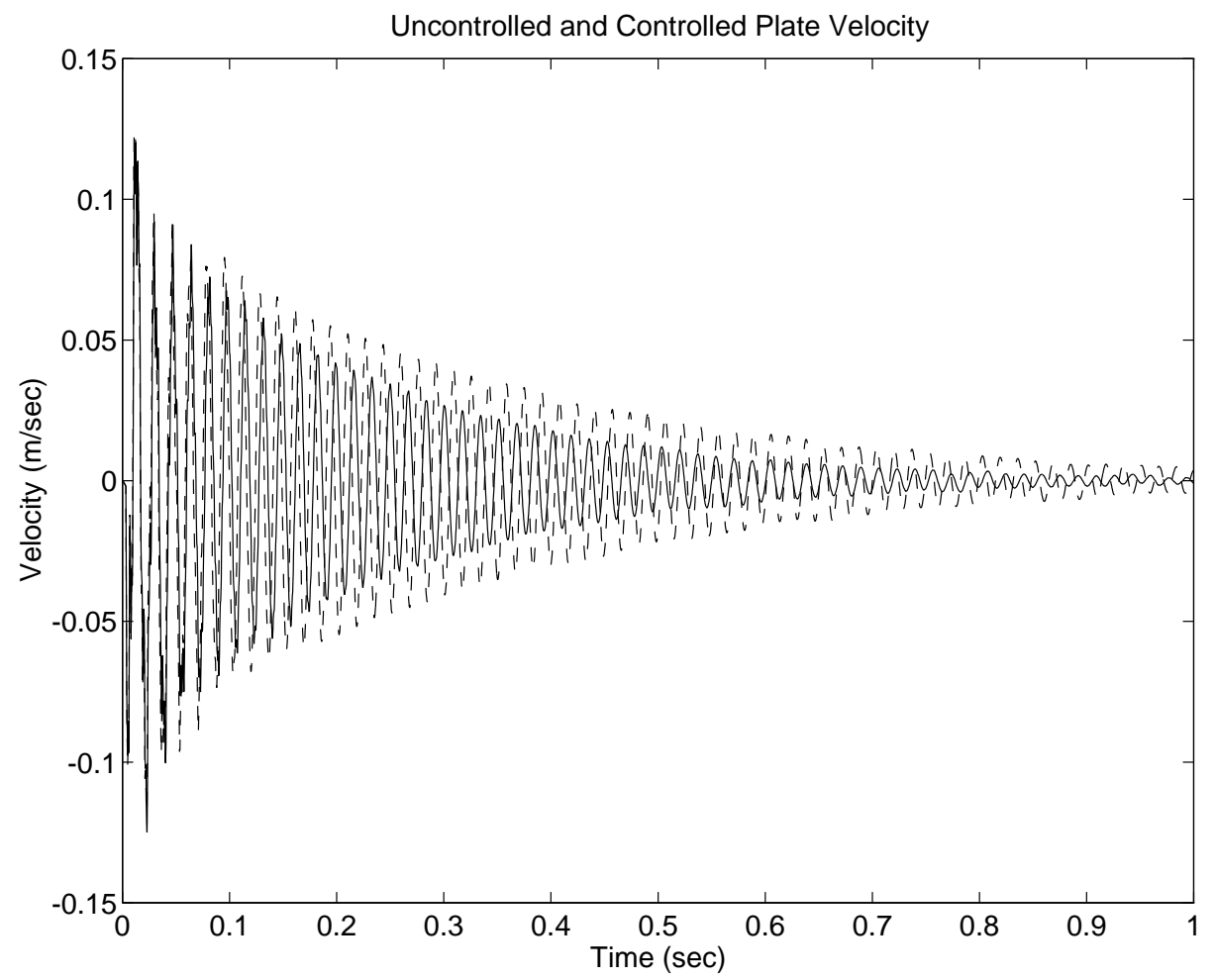

Fig. 4. Uncontrolled and controlled plate vibrations in response to an impact hammer hit.

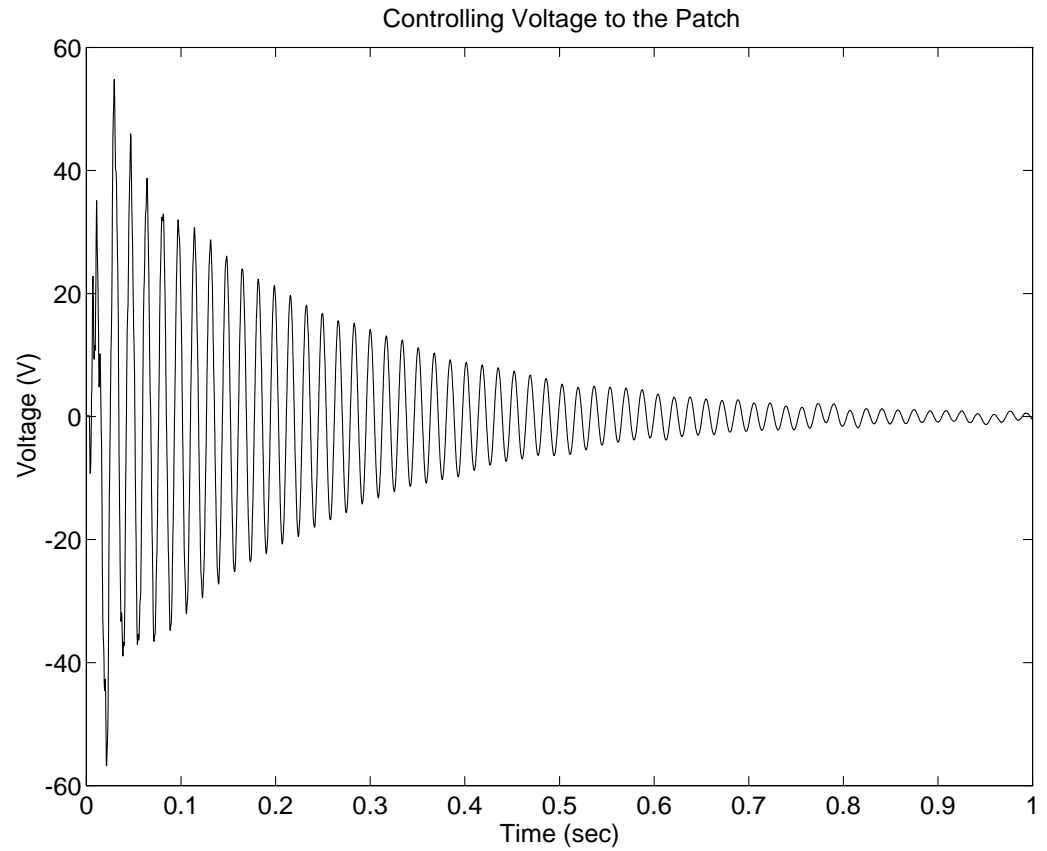

Fig. 5. The controlling voltage determined from (7). 


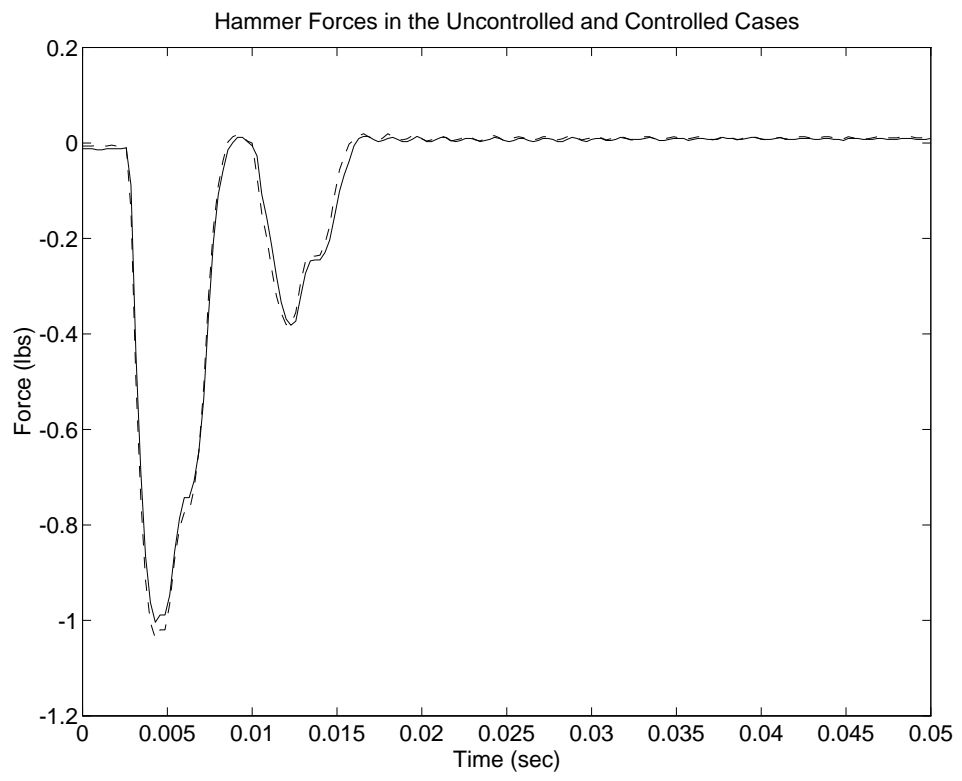

Fig. 6. The force delivered by the impact hammer in the uncontrolled and controlled cases, - - - (Controlled Case), --- (Uncontrolled Case).

\section{CONCLUDING REMARKS}

In this paper, the experimental implementation of a PDE-based parameter estimation and feedback control strategy for structural and structural acoustic systems has been considered. The structure under consideration was a thin circular plate with a centered piezoceramic patch. After modeling the structure by thin plate equations with piecewise constant parameters (to account for the material variation in the region of the patch), the problem was discretized using a Fourier-Galerkin basis to obtain a form that was useful for implementing a feedback control scheme. The first experimental step was to then estimate the physical plate and patch parameters through a data fitting technique. With the estimated parameters in the system model, output feedback control results were used to obtain a feedback law which provided a voltage to be applied to the patches. When experimentally implemented, this control technique led to a reduction in the plate vibrations. While the implementation procedures are not yet optimal, this provided a test as to the feasibility of implementing a PDE-based method in this manner as well as indicated directions for further experimental research with both structural and structural acoustic systems.

\section{References}

[1] H.T. Banks and J.A. Burns, Introduction to Control of Distributed Parameter Systems, Birkhäuser, to appear.

[2] H.T. Banks, W. Fang, R.J. Silcox and R.C. Smith, "Approximation methods for control of acoustic/structure models with piezoceramic actuators," J. Intell. Mat. Sys. Structures, 4(1), pp. 98-116, 1993.

[3] H.T. Banks, K. Ito and B.B. King, "Theoretical and computational aspects of feedback in structural systems with piezoceramic controllers," in Computation and Control III, Proc. Third Bozeman Conf., Bozeman, MT, 1992, Progress in Systems and Control Theory, Vol. 15, Birkhäuser Boston, Inc., pp. 1-27, 1993.

[4] H.T. Banks, K. Ito and Y. Wang, "Computational methods for identification and feedback control in structures with piezoceramic actuators and sensors," in Recent Advances in Adaptive and Sensor Materials and their Applications, (C.A. Rogers and R.C. Rogers, ed.), Technomic Publ., pp. 111-119, 1992; J. Intell. Mat. Sys. Structures, 4, pp. 469-476, 1993. 
[5] H.T. Banks, R.J. Silcox and R.C. Smith, "The modeling and control of acoustic/structure interaction problems via piezoceramic actuators: 2-D numerical examples," to appear in ASME Journal of Vibration and Acoustics.

[6] R.A. Burdisso and C.R. Fuller, "Dynamic behavior of structural-acoustic systems in feedforward control of sound radiation," J. Acoust. Soc. Am., 92(1), pp. 277-286, 1992.

[7] E.K. Dimitriadis and C.R. Fuller, "Active control of sound transmission through elastic plates using piezoelectric actuators," AIAA $12^{\text {th }}$ Aeroacoustics Conf. (San Antonio), April 10-12, Paper 89-1062, 1989.

[8] J.J. Dosch, D.J. Inman and E. Garcia, "A self-sensing piezoelectric actuator for collocated control," Journal of Intelligent Material Systems and Structures, 3, pp. 166-185, 1992.

[9] C.R. Fuller, C.H. Hansen and S.D. Snyder, "Active control of structurally radiated noise using piezoceramic actuators," Proc. of Inter-Noise 89 (Newport Beach, CA), 509-512, 1989.

[10] C.R. Fuller, S.D. Snyder, C.H. Hansen and R.J. Silcox, "Active control of interior noise in model aircraft fuselages using piezoceramic actuators," AIAA $13^{\text {th }}$ Aeroacoustics Conf. (Tallahassee) October 22-24, Paper 90-3922, 1990 .

[11] H.T. Banks, Y. Wang and D.J. Inman, "Bending and shear damping in beams: frequency domain estimation techniques," to appear in Journal of Vibration and Acoustics.

[12] H.T. Banks, Y. Wang, D.J. Inman and J.C. Slater, "Approximation and parameter identification for damped second order systems with unbounded input operators," Center for Research in Scientific Computation Technical Report, CRSC-TR93-9, North Carolina State University, submitted to Control: Theory and Advanced Technology.

[13] H.T. Banks, S.S. Gates, I.G. Rosen and Y. Wang, "The identification of a distributed parameter model for a flexible structure," SIAM J. Control and Opt., 26, pp. 743-762, 1988.

[14] H.T. Banks, R.J. Silcox and R.C. Smith, "Numerical simulations of a coupled 3-D structural acoustics system," in the Proc. of the Second Conf. on Recent Advances in Active Control of Sound and Vibration, Blacksburg, VA, pp. 85-97, 1993.

[15] H.T. Banks and R.C. Smith, "Modeling and approximation of a coupled 3-D structural acoustics problem," in Computation and Control III, Proc. Third Bozeman Conf., Bozeman, MT, 1992, Progress in Systems and Control Theory, Vol. 15, Birkhäuser Boston, Inc., pp. 29-48, 1993.

[16] H.T. Banks, R.C. Smith and Y. Wang, "The modeling of piezoceramic patch interactions with shells, plates, and beams," ICASE Report 92-66, to appear in Quart. App. Math..

[17] T. Basar and P. Bernhard, $H^{\infty}$ - Optimal Control and Related Minimax Design Problems, Birkhäuser, Boston, 1991.

[18] W.L. Hallauer, Jr. and S.E. Lamberson, "Experimental active vibration damping of a plane truss using hybrid actuation," Proc. 30th AIAA/ASME/ASCE/AHS/ASC Structural Dynamics and Materials Conf., Paper 891169-CP, pp. 80-90, 1989. 